\title{
Cerebrovascular reactivity changes in acute concussion: a controlled cohort study
}

\author{
Runrun Wang ${ }^{1,2,3}$, Julien Poublanc ${ }^{1}$, Adrian P. Crawley ${ }^{1}$, Olivia Sobczyk ${ }^{1}$, Sander Kneepkens ${ }^{1}$, \\ Larissa Mcketton $^{1}$, Charles Tator ${ }^{4}$, Renhua $\mathrm{Wu}^{3}$, David J. Mikulis ${ }^{1}$ \\ ${ }^{1}$ Joint Department of Medical Imaging, University Health Network, The Toronto Western Hospital, The University of Toronto, Toronto, Ontario, \\ Canada; ${ }^{2}$ Department of Neurology, Henan Provincial People's Hospital, Zhengzhou University People's Hospital, Henan, China; ${ }^{3}$ Department \\ of Medical Imaging, the Second Affiliated Hospital, Medical College of Shantou University, Shantou, China; ${ }^{4}$ Department of Surgery, Division of \\ Neurosurgery, University Health Network, The Toronto Western Hospital, The University of Toronto, Toronto, Ontario, Canada
}

Contributions: (I) Conception and design: R Wang, R Wu, DJ Mikulis; (II) Administrative support: J Poublanc; (III) Provision of study materials or patients: AP Crawley, O Sobczyk, S Kneepkens; (IV) Collection and assembly of data: L Mcketton, C Tator; (V) Data analysis and interpretation: R Wang, J Poublanc; (VI) Manuscript writing: All authors; (VII) Final approval of manuscript: All authors.

Correspondence to: Dr. David J. Mikulis. Joint Department of Medical Imaging, 3MC-431, Toronto Western Hospital, 399 Bathurst Street, Toronto, Ontario M5T 2S8, Canada. Email: David.Mikulis@uhn.ca; Dr. Renhua Wu. Department of Medical Imaging, The Second Affiliated Hospital, Medical College of Shantou University, Shantou 515041, China. Email: rhwu@stu.edu.cn.

Background: Evidence suggests that cerebrovascular reactivity (CVR) increases within the first week after the incidence of concussion, indicating a disruption of normal autoregulation. We sought to extend these findings by investigating the effects of acute concussion on the speed of CVR response and by visualizing global and regional impairments in individual patients with acute concussion.

Methods: Twelve patients aged 18-40 years who experienced concussion less than a week before this prospective study were included. Twelve age and sex-matched healthy subjects constituted the control group. In all subjects, CVR was assessed using blood oxygenation level-dependent (BOLD) echo-planar imaging with a 3.0T MRI scanner, in combination with changes in end-tidal partial pressure of $\mathrm{CO}_{2}\left(\mathrm{P}_{\mathrm{ET}} \mathrm{CO}_{2}\right)$. In each subject, we calculated the CVR amplitude and CVR response time in the gray and white matter using a step and ramp $\mathrm{P}_{\mathrm{ET}} \mathrm{CO}_{2}$ challenge. In addition, a separate group of 39 healthy controls who underwent the same evaluation was used to create atlases with voxel-wise mean and standard deviation of CVR amplitude and CVR response time. This allowed us to convert each metric of the 12 patients with concussion and the 12 healthy controls into z-score maps. These maps were then used to generate and compare z-scores for each of the two groups. Group differences were calculated using an unpaired $t$-test.

Results: All studies were well tolerated without any serious adverse events. Anatomical MRI was normal in all study subjects. No differences in $\mathrm{CO}_{2}$ stimulus and $\mathrm{O}_{2}$ targeting were observed between the two participant groups during BOLD MRI. With regard to the gray matter, the CVR magnitude step $(\mathrm{P}=0.117)$ and $\operatorname{ramp}+10(\mathrm{P}=0.085)$ were not significantly different between patients with concussion and healthy controls. However, the tau value was significantly lower in patients with concussion than in the healthy controls $(\mathrm{P}=0.04)$. With regard to the white matter, the CVR magnitude step $(\mathrm{P}=0.003)$ and ramp $+10(\mathrm{P}=0.031)$ were significantly higher and the tau value $(\mathrm{P}=0.024)$ was significantly shorter in patients with concussion than in healthy controls. After $\mathrm{z}$-score transformation, the $\mathrm{z}$ tau value was significantly lower in patients with concussion than in healthy controls (Grey matter $\mathrm{P}=0.021$, White matter $\mathrm{P}=0.003$ ). Comparison of the three parameters, $\mathrm{z}$ ramp +10 , $\mathrm{z}$ step, and $\mathrm{z}$ tau, between the two groups showed that $\mathrm{z}$ step (Grey matter $\mathrm{P}=0.035$, White matter $\mathrm{P}=0.005$ ) was the most sensitive parameter and that $\mathrm{z}$ ramp +10 (Grey matter $\mathrm{P}=0.073$, White matter $\mathrm{P}=0.126$ ) was the least sensitive parameter.

Conclusions: Concussion is associated with patient-specific abnormalities in BOLD cerebrovascular 
responsiveness that occur in the setting of normal global CVR. This study demonstrates that the measurement of CVR using BOLD MRI and precise $\mathrm{CO}_{2}$ control is a safe, reliable, reproducible, and clinically useful method for evaluating the state of patients with concussion. It has the potential to be an important tool for assessing the severity and duration of symptoms after concussion.

Keywords: Concussion; blood oxygen level-dependent imaging; cerebrovascular reactivity; cerebral blood flow $(\mathrm{CBF})$

Submitted Nov 23, 2020. Accepted for publication Jun 18, 2021.

doi: 10.21037/qims-20-1296

View this article at: https://dx.doi.org/10.21037/qims-20-1296

\section{Introduction}

A non-blast-related concussion is a form of brain injury caused by either a direct blow to the head or an indirect injury in which force generated via impact to another part of the body is transmitted to the head (1). Mild traumatic brain injury (mild TBI) and concussion are used almost interchangeably (2). Thereby, we did not distinguish between concussion and mild/moderate TBI. Concussion results in a disturbance of brain function and is associated with a number of symptoms, including headaches, dizziness, verbal and visual impairment, fatigue, and poor concentration (3). Symptoms usually resolve spontaneously, but sometimes last for days, weeks, or even longer (4). With regard to concussion, our understanding of the pathophysiology that follows and the relationship with the occurrence and persistence of symptoms is limited (4). Furthermore, because of a lack of visible brain injury on conventional CT or MR images, the application of these techniques for single-subject diagnosis has not been achieved. Using advanced neuroimaging techniques can help distinguish a merged group of individuals with concussion from a merged group of controls (5). Therefore, it is important to develop a reproducible and objective diagnostic imaging biomarker for reliable detection of concussion in individual patients and for the prediction of recovery in concussed patients.

Concussion imaging research mainly focuses on neuronal damage with a lesser emphasis on vascular dysfunction. However, blood flow metrics may be more sensitive than measurements of neuronal integrity (6). It is known that the cerebral blood flow (CBF), vascular reserve, and blood-brain barrier integrity are altered, implying structural/functional dysfunction of the neuro-glio-vascular unit (7). The diagnosis of concussion majorly depends on the scale and main complaint of the patient; however, there is a lack of objective or quantitative diagnostic methods for concussion. In recent research, several advanced neuroimaging techniques have shown significant differences in white matter anisotropy, brain activation patterns, and resting $\mathrm{CBF}$ between a concussion patient group and a healthy control group (8). Although these studies have promoted our understanding of the impact of concussions on brain structure and function, the detection of differences in neuroimaging biomarkers in each group may not accurately reflect the pathophysiological mechanism behind the symptoms and injury status of individual patients with concussions. Cerebrovascular reactivity (CVR) reflects the ability of the vasculature to accommodate changes in blood flow demand; thus, it serves as a critical imaging tool for mapping the vascular reserve (9). It has long been used to clarify the underlying brain vascular physiology and pathophysiology in the presence of neurovascular diseases (10). Changing the arterial carbon dioxide partial pressure $\left(\mathrm{PaCO}_{2}\right)$ and the actual vasoactive stimulus in a controlled manner is progressively more popular in non-invasive CVR studies (11). For clinicians and researchers interested in the role of $\mathrm{CBF}$ regulation in the pathophysiology of concussion, it is important to be able to assess the significance of changes in blood flow in the brain after a concussion. In the most common non-invasive MRI sequence, blood oxygen level dependence (BOLD) and arterial spin labeling (ASL) are used to evaluate CBF. The relationship between the BOLD signal and CBF is quite linear (12); the BOLD signal can be used as a substitute for $\mathrm{CBF}$ measurements (13). ASL is a technique that can directly measure $\mathrm{CBF}$ in brain tissue. However, the narrowing of blood vessels may increase the transit time, leading to the delayed arrival of the labeled protons, thereby causing errors in CBF measurement (14).

Controlled $\mathrm{PaCO}_{2}$ in BOLD magnetic resonance imaging (MRI), which measures $\mathrm{CBF}$ with a high spatial and temporal 
resolution, has become increasingly popular in non-invasive CVR research (15). The $\mathrm{CO}_{2} \mathrm{CVR}$ and CBF of BOLD MRI are very sensitive to small changes in $\mathrm{PaCO}_{2}$. Besides efforts to accurately measure $\mathrm{PaCO}_{2}, \mathrm{CVR}$ can be standardized further by the BOLD signal for actual changes in end-tidal partial pressure of $\mathrm{CO}_{2}\left(\mathrm{P}_{\mathrm{ET}} \mathrm{CO}_{2}\right) \cdot \mathrm{CO}_{2}$ change stimulus affects the vascular response, thereby affecting the BOLD signal. All these characteristics of the stimuli need to be standardized to eliminate noise that introduces variability and produces comparable data between subjects and studies (16). The pathophysiology of concussion in humans is associated with microstructural perturbations including axonopathy, astrocytosis, neuroinflammation, and microvascular injury (16). The physiopathological change after brain concussion is the rapid release of neurotransmitters, which can cause ion imbalance across the neuron membrane. Reestablishing ion homeostasis consumes energy and causes dynamic changes in the brain glucose uptake. The extent and duration of these changes are related to the severity of the injury, and mild injuries show faster normalization. Sex differences in the brain further increase the performance of patients with concussion (17). One study compared the relative CBF graphs of the controlled assessment of 24 concussion contact sports athletes obtained 24-48 hours after injury with the control group of 24 paired contact sports athletes. CBF detected in several brain regions of concussion athletes was significantly reduced (18). However, research has not yet addressed whether the resting flow disturbances are mediated by defects in neurogliovascular coupling or by direct injury to blood vessels. Human brain function mainly depends on continuous oxygen delivery and active regulation of metabolic nutrition CBF. The cerebrovascular function is accurately titrated through various physiological mechanisms and is characterized by complex integration. Although there is evidence that $\mathrm{CBF}$ regulation during exercise depends on changes in blood pressure, neurogenic activity, and cardiac output, their role as the main regulator of CBF on exercise response remains controversial. In contrast, the balance between the partial pressure of carbon dioxide and brain metabolism continues to gain empirical support (19). When arterial carbon dioxide stimuli are tightly controlled and applied during blood flow mapping using MRI, measurement of the speed and magnitude of the vasodilatory response, i.e., CVR, is accurate and reproducible (20-22), and thus, ideally suited for this purpose.

\section{Methods}

\section{Subjects and ethical approval}

Participants were recruited in cooperation with the Hull-Ellis Concussion and Research Clinic. This study conformed to the standards set by the latest revised version of the Declaration of Helsinki (as revised in 2013) and was approved by the research ethics board of the University Health Network.

A prospective cohort of 12 subjects who experienced concussion less than one week before the commencement of the study and 12 age- and sex-matched controls were recruited. Inclusion criteria for this study were (I) age of 1840 years; (II) MRI scans obtained $1-7$ days (median $=4$ days) from the time of injury; and (III) an initial Glasgow coma scale (GCS) score of 13-15; (IV) observation of any period of loss of consciousness less than 30 minutes or any posttraumatic amnesia less than 24 hours, or record of change in mental status (confused, disoriented, or dazed); (V) clinical diagnosis of concussion by a neurosurgeon. Fiftyone individuals without a history of (I) concussion or brain injury, (II) cerebrovascular disease, and (III) either remote or current neurological condition were recruited as healthy control subjects and their BOLD CVR images were collected. The following were the exclusion criteria: (I) age $<18$ years old, $>40$ years old; (II) MRI examination after 1 week of onset; (III) pregnancy, medically documented history of previous brain injury, neurological disorders, or psychoactive medications; (IV) CT indication of any metal in the brain and body or known contraindication to MRI (such as a pacemaker or other non-MR compatible implanted device) as identified by safety screening; (V) drug addiction leading to cognitive changes; (VI) radiological examinations indicating intracranial lesions (tumor, hemorrhage, parasites, etc.); (VII) MRI data of the subject with head motion and artifacts; (VIII) and unusable data. A summary of participant characteristics can be found in Table 1. Each healthy control's age = age of a patient with concussion \pm 2 years. Among these controls, 12 sexmatched controls were used for comparison with the group of patients with concussion, and 39 were used to generate atlases for each of the following three metrics: CVR magnitude step, CVR magnitude ramp +10 , and CVR time response (tau). All patients provided written informed consent prior to study participation. 
Table 1 Summary of participant characteristics

\begin{tabular}{lccc}
\hline Patient number & CP-age (years) & Sex & HC-age (years) \\
\hline 01 & 39 & $\mathrm{~F}$ & 38 \\
02 & 32 & $\mathrm{M}$ & 30 \\
03 & 40 & $\mathrm{M}$ & 39 \\
04 & 34 & $\mathrm{~F}$ & 33 \\
05 & 18 & $\mathrm{~F}$ & 20 \\
06 & 29 & $\mathrm{~F}$ & 29 \\
07 & 22 & $\mathrm{~F}$ & 22 \\
08 & 31 & $\mathrm{M}$ & 30 \\
09 & 29 & $\mathrm{M}$ & 29 \\
10 & 24 & $\mathrm{M}$ & 24 \\
11 & 28 & $\mathrm{~F}$ & 28 \\
12 & 40 & $\mathrm{M}$ & 39 \\
\hline
\end{tabular}

$\mathrm{CP}$, patients with concussion; $\mathrm{HC}$, healthy controls.

\section{$\mathrm{P}_{E T} \mathrm{CO}_{2}$ recording}

In all subjects, changes in $\mathrm{P}_{\mathrm{ET}} \mathrm{CO}_{2}$ were achieved by precise delivery of $\mathrm{CO}_{2}$ via a face mask, using a sequential gas delivery breathing circuit connected to an automated gas blender (23) (Respir ${ }^{\text {Act }}$, Thornhill Research Inc., Toronto, ON, Canada). Before MRI assessment, all subjects were familiarized with the test by exposing them to hypercapnia for $1 \mathrm{~min}$. The $\mathrm{P}_{\mathrm{ET}} \mathrm{CO}_{2}$ stimulus protocol was defined by the following sequence (Figure 1): normocapnia for $120 \mathrm{~s}$ at resting $\mathrm{P}_{\mathrm{ET}} \mathrm{CO}_{2}$, hypercapnia for $120 \mathrm{~s}$ at resting $\mathrm{P}_{\mathrm{ET}} \mathrm{CO}_{2}+$ $10 \mathrm{mmHg}$, and normocapnia for $120 \mathrm{~s}$ at resting $\mathrm{P}_{\mathrm{ET}} \mathrm{CO}_{2}$. $\mathrm{P}_{\mathrm{ET}} \mathrm{CO}_{2}$ was then slowly reduced to the baseline $\mathrm{P}_{\mathrm{ET}} \mathrm{CO}_{2}-$ $10 \mathrm{mmHg}$ level by hyperventilation over $60 \mathrm{~s}$, followed by a slow ramp increase to the baseline $\mathrm{P}_{\mathrm{ET}} \mathrm{CO}_{2}+15 \mathrm{mmHg}$ level over 240 s. Finally, $\mathrm{P}_{\mathrm{ET}} \mathrm{CO}_{2}$ was returned to the resting baseline level for $120 \mathrm{~s}$. This device allows the precise manipulation of $\mathrm{P}_{\mathrm{ET}} \mathrm{CO}_{2}$ levels under isoxic conditions (24).

B
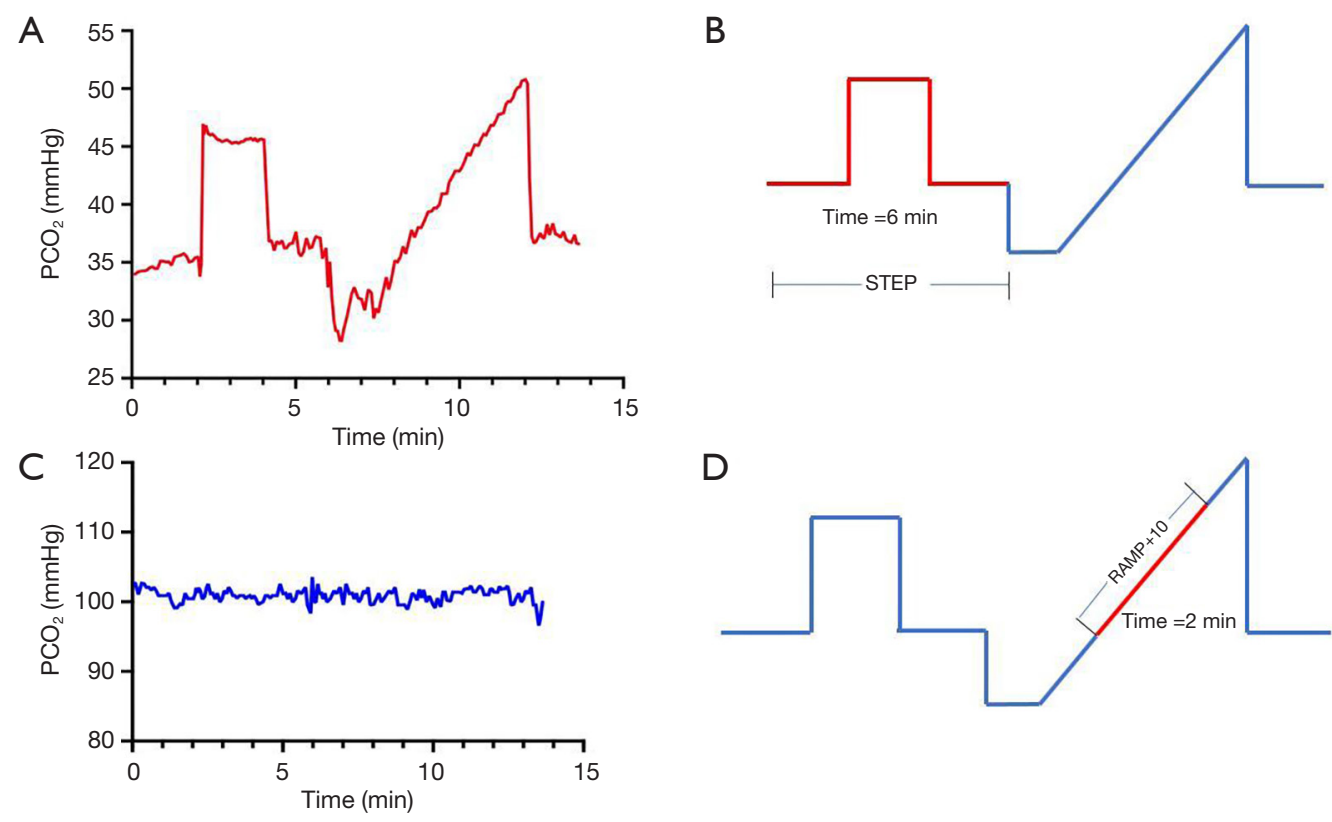

Figure 1 Example of the end-tidal gas targeting for $\mathrm{CO}_{2}(\mathrm{~A})$ and $\mathrm{O}_{2}(\mathrm{C})$ in a patient. The analysis was separated into step (B) and ramp + 10 (D) segments. In (D), the red section starts at the patient's resting end-tidal partial pressure of $\mathrm{CO}_{2}\left(\mathrm{PETCO}_{2}\right)$ and ends 10 mmHg above it; hence, the name ramp +10 . 
During the application of this protocol, blood pressure was recorded approximately every $2 \mathrm{~min}$.

\section{MRI}

All images were acquired on a $3 \mathrm{~T}$ scanner using an eightchannel phased-array receiver coil (Signa; GE Healthcare, Milwaukee, Wisconsin). T1-weighted high-resolution images of $1 \mathrm{~mm}$ isotropic voxels were acquired for re-registration with BOLD CVR, as well as for spatial normalization and tissue segmentation into gray and white matter.

During the application of the $\mathrm{CO}_{2}$ stimulus protocol, BOLD CVR images were acquired with an echo-planar imaging gradient sequence (Repetition time/Echo time $=2,400 / 30 \mathrm{~ms}$, matrix size $=64 \times 64$, isotropic voxel size $=3.5 \mathrm{~mm}$, and flip angle $=85^{\circ}$ ).

\section{Analysis}

\section{$\mathrm{P}_{\mathrm{ET}} \mathrm{CO}_{2}$ and statistics}

First, $\mathrm{P}_{\mathrm{ET}} \mathrm{CO}_{2}$ was time-shifted to approximately match the average BOLD signal. A correlation was calculated between $\mathrm{P}_{\mathrm{ET}} \mathrm{CO}_{2}$ and all voxels of the brain. Second, "Healthy" voxel time series with correlations exceeding 0.7 were averaged together and used as a reference to re-adjust $\mathrm{P}_{\mathrm{ET}} \mathrm{CO}_{2}$. Finally, $\mathrm{P}_{\mathrm{ET}} \mathrm{CO}_{2}$ was re-sampled to match the time resolution of the BOLD signal. Multivariate regression analysis and covariate-adjusted analysis of variance were used for the statistical analysis. Baseline $\mathrm{P}_{\mathrm{ET}} \mathrm{CO}_{2}$ was a covariate in the covariate-adjusted analysis. Multivariate regression analysis was used to compare the differences in CVR indicators between the patient and the control group to correct for age and sex.

\section{CVR magnitude step}

The raw BOLD signal was first linearly detrended and scaled, such that the mean baseline raw signal was 100 . The CVR magnitude step metric was obtained by calculating the slope of linear regression of the BOLD signal with $\mathrm{P}_{\mathrm{ET}} \mathrm{CO}_{2}$ over the step section of the protocol (Figure 1B). CVR magnitude step was therefore expressed as \%BOLD per $\mathrm{mmHg}$. The calculation was repeated for all voxels of the brain to generate a map (Figure 2).

\section{CVR magnitude ramp + 10}

This metric was obtained using the same method as above over the ramp section of the protocol (Figure 1D). This section started at the patient's own resting $\mathrm{P}_{\mathrm{ET}} \mathrm{CO}_{2}$ and ended $10 \mathrm{mmHg}$ above it. It was therefore named ramp + 10. The associated maps are shown in Figure 2.

\section{CVR time response, tau}

Tau is a measure of the inverse speed of CVR response. Short tau is equivalent to fast responses and long tau to slow responses. This metric was determined by the linear regression of the $\mathrm{BOLD}$ signal using $\mathrm{P}_{\mathrm{ET}} \mathrm{CO}_{2}$ convolved with an exponential function over the step section of the protocol (Figure 1B). The time characteristic, tau, of the exponential that best fits the BOLD signal is a measure of CVR response time. This method is explained in further detail elsewhere (25). Note that the tau values of voxels with negative amplitudes experiencing a steal phenomenon are in fact governed by healthy positive voxels. Because those time responses do not represent the response of the local vasculature, their associated voxels were removed in the subsequent steps of the analysis. The maps are shown in Figure 2.

\section{Spatial normalization}

The high-resolution T1-weighted volume was segmented and spatially normalized using spm8 (26). The normalization was achieved by re-registration to the Montreal Neurological Institute (MNI) atlas using an affine followed by non-linear re-registration. The segmentation provided probability maps of the gray matter, white matter, and cerebrospinal fluid. The calculated transformation matrix was then applied to the CVR maps in order to combine multiple subjects.

\section{$z$ maps}

For each metric, the atlas was defined as a map of the mean $(\mu)$ and a map of the standard deviation $(\sigma)$ across the 39 normalized healthy controls. These atlases were subsequently used to calculate voxel-wise $\mathrm{z}$-scores using the equation $\mathrm{z}=(\mathrm{x}-\mu) / \sigma$, where $\mathrm{x}$ is one of the 3 metrics (Figure 3).

\section{Brain average and z-score}

A comparison of individual results to z-score atlases of controls can potentially provide individual subject diagnostic capability (27). Z-score is a very important statistical parameter. When the distribution form of the original score is normal, all the original scores are transformed into z-scores, and the standard normal distribution is achieved. The application of z-score is mainly to express the relative position of each original data. It could express the 


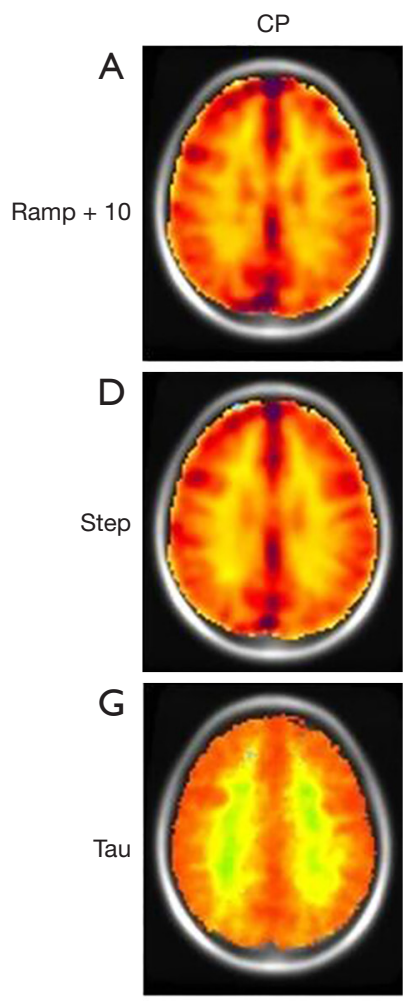

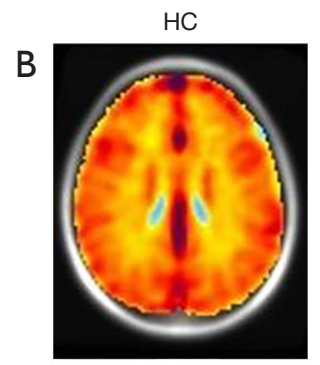

E
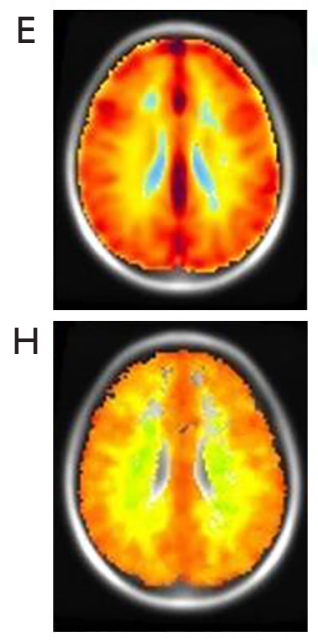
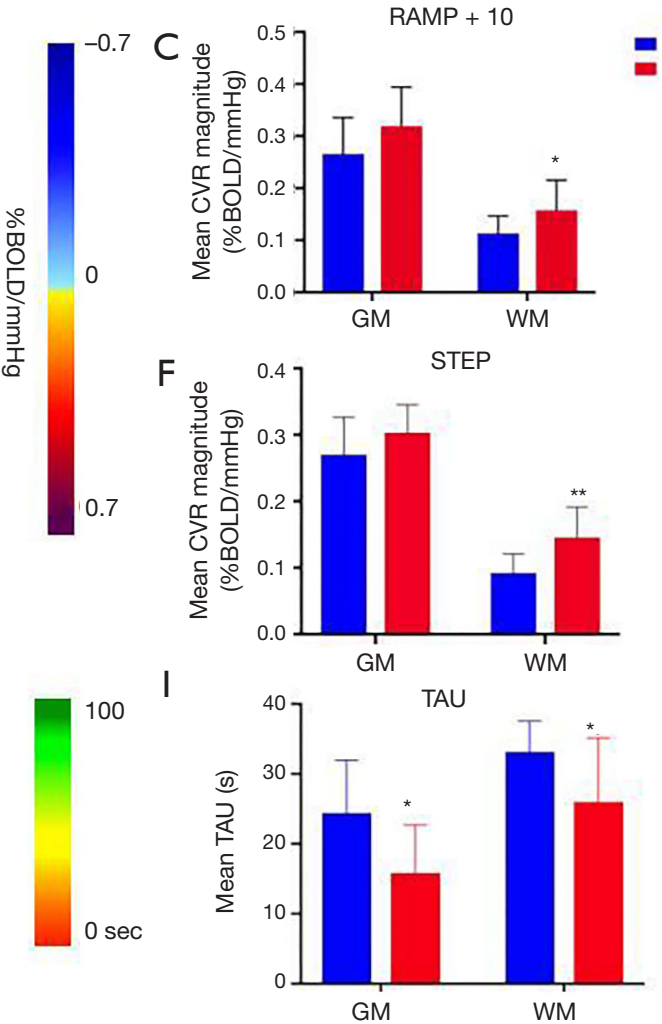

Figure 2 Mean maps of patients with concussion and healthy controls for CVR ramp + 10 (A and B), CVR step (D and E), and CVR tau (G and H). Note that some areas in the CVR tau map (h) are masked out because of negative CVR amplitudes. C, F, and I represent the mean CVR ramp + 10, CVR step, and CVR tau in patients with concussion and healthy controls, for the gray and white matter separately. A $t$-test was performed to compare differences in the above metrics between patients with concussion and healthy controls $\left({ }^{*}, \mathrm{P}<0.05 ;{ }^{* *}, \mathrm{P}<0.005\right)$. CVR, cerebrovascular reactivity; BOLD, blood oxygenation level dependence; CP, patients with concussion; HC, healthy controls; GM, gray matter; WM, white matter.

proportion of data below or above the mean. $\mathrm{z}=-2$ and $\mathrm{z}=2$ indicate that the total score is two standard deviations from the top to the bottom.

The average CVR magnitude ramp +10 , CVR magnitude step, and CVR response time tau of the gray and white matter were extracted for each of the 12 healthy controls and 12 patients with concussion. Group differences were calculated using an unpaired $t$-test. The same process was repeated using $\mathrm{z}$-scores of these three metrics.

\section{Results}

\section{Study tolerability}

All study participants successfully completed the full imaging study without any serious adverse events.

\section{Original metrics}

Mean maps for CVR magnitude ramp + 10, CVR magnitude step, and CVR response time (tau) corresponding to patients with concussion and healthy controls are shown in Figure 2. With regard to the gray matter, the CVR magnitude step $(\mathrm{P}=0.117)$ and ramp + $10(\mathrm{P}=0.085)$ were not significantly different between patients with concussion and healthy controls. However, the tau value was significantly lower in patients with concussion than in healthy controls $(\mathrm{P}=0.004)$. With regard to the white matter, we found that the CVR magnitude step $(\mathrm{P}=0.003)$ and $\mathrm{ramp}+10(\mathrm{P}=0.031)$ were significantly higher and the tau $(\mathrm{P}=0.024)$ was significantly shorter in patients with concussion than in healthy controls. 

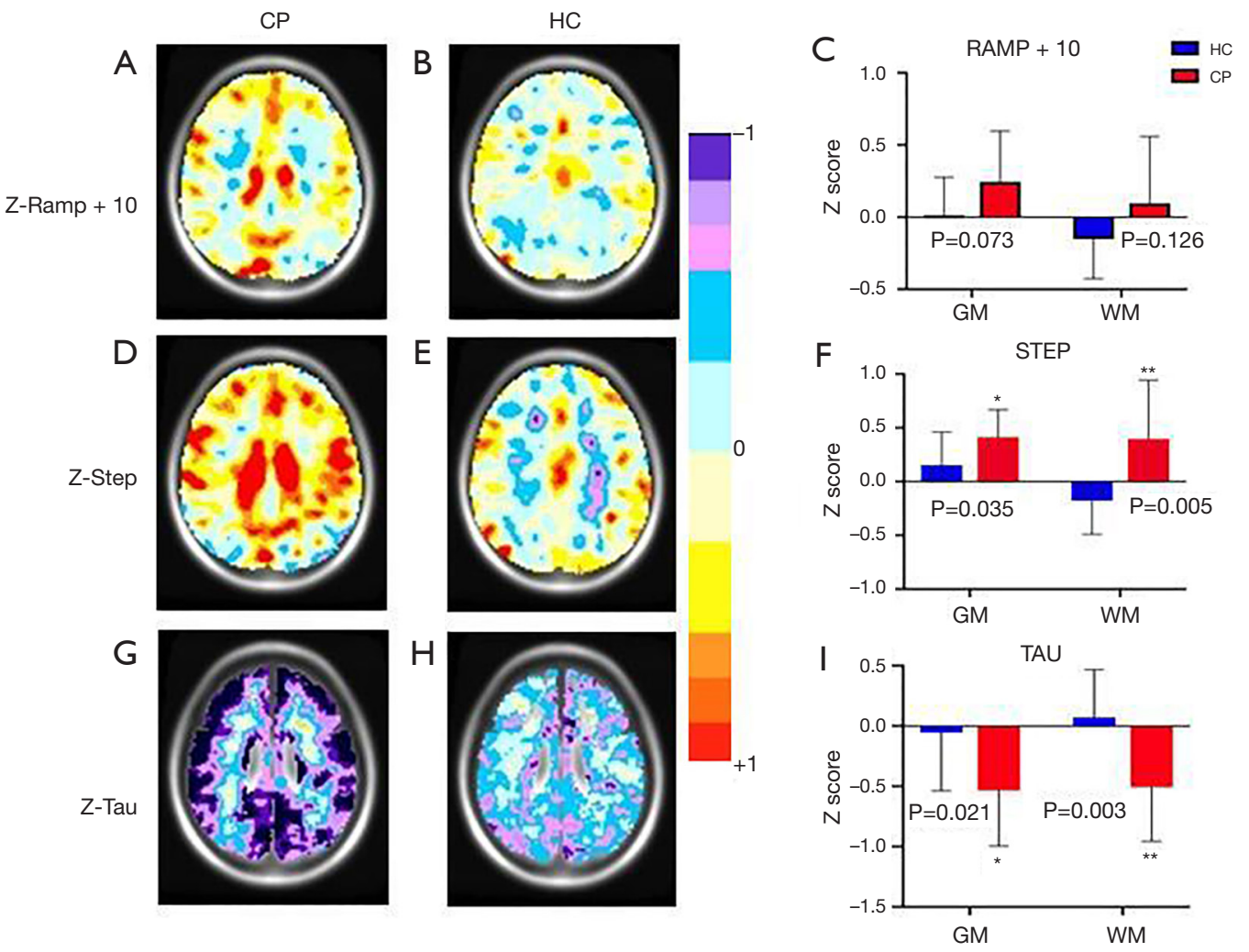

Figure 3 The mean maps of patients with concussion and healthy controls for $\mathrm{z}$ ramp + 10 (A and B), z step (D and E), and z tau (G and H). $\mathrm{C}, \mathrm{F}$, and I represent the mean $\mathrm{z}$ ramp +10 , z step, and $\mathrm{z}$ tau in patients with concussion and healthy controls, for the gray and white matter separately. A $t$-test was performed to compare the differences in the above metrics between patients with concussion and healthy controls $\left(^{*}\right.$, $\mathrm{P}<0.05$; $\left.{ }^{* *}, \mathrm{P}<0.005\right)$. CP, patients with concussion; HC, healthy controls; GM, gray matter; WM, white matter.

\section{$z$-scores of the metrics}

Figure 3 shows the means of the $\mathrm{z}$ step, $\mathrm{z}$ ramp +10 , and $\mathrm{z}$ tau for patients with concussion and healthy controls. This figure also shows that after $\mathrm{z}$-score transformation, the $\mathrm{z}$ step values corresponding to the gray matter and white matter were significantly higher and lower (Grey matter $\mathrm{P}=0.035$, White matter $\mathrm{P}=0.005$ ), respectively, in patients with concussion than in healthy controls. We observed the same trend for $\mathrm{z}$ tau (Grey matter $\mathrm{P}=0.021$, White matter $\mathrm{P}=0.003$ ) and $\mathrm{z}$ ramp + 10 , but the differences were not significant for $\mathrm{z}$ ramp +10 in either the gray matter or white matter (Grey matter $\mathrm{P}=0.073$, White matter $\mathrm{P}=0.126$ ). The subjects with acute concussion exhibited significantly larger and faster CVR responses than the healthy controls, especially in the white matter.

\section{z-score analysis}

The z-scores were calculated to provide more detailed results. Using z-score analysis and processing software (SMP8; Department of Imaging Neuroscience, London), each individual brain volume was co-registered from the normative data into the MNI standard space. The CVR value of each subject was scored by assigning z-scores according to the average and SD values of the corresponding voxels of the map, and then each z-score was calculated based on the voxel numbers. $z=-2$ refers to the comparison of voxel numbers that have $z$-scores of -2 . The coregistered T1-weighted anatomical images were segmented into gray and white matter using the aforementioned SMP8 software. For each subject, the average z-scores of the gray and white matter were calculated. Table 2 shows the differences between the patients with concussion and healthy controls. Comparison of the three parameters, $z$ ramp $+10, z$ step, $z$ tau, between the two groups showed that $\mathrm{z}$ step was the most sensitive parameter and that $\mathrm{z}$ ramp +10 was the least sensitive parameter. With regard to 
Table 2 Differences in z-scores between patients with concussion and healthy controls

\begin{tabular}{|c|c|c|c|c|c|c|}
\hline Z-scores & \multicolumn{2}{|c|}{ Ramp + 10} & \multicolumn{2}{|c|}{ Step } & \multicolumn{2}{|c|}{ Tau } \\
\hline$Z=+2$ & 0.087 & 0.050 & 0.140 & $0.010^{*}$ & 0.696 & 0.896 \\
\hline$Z=-2$ & 0.568 & 0.471 & 0.342 & $0.024^{\star}$ & $0.017^{\star}$ & $0.005^{*}$ \\
\hline Mean & 0.214 & 0.387 & $0.028^{*}$ & $0.001^{\star *}$ & 0.122 & $0.032^{*}$ \\
\hline
\end{tabular}

${ }^{*}, \mathrm{P}<0.05 ;{ }^{*}, \mathrm{P}<0.005$. GM, gray matter; WM, white matter.

z-scores for the white matter and gray matter, z ramp +10 scores did not show marked difference either when $\mathrm{z}=-2$ or when $\mathrm{z}=2$, but this difference is significantly amplified for $\mathrm{z}$ tau when $z=-2$. The largest difference between the patient and control groups was seen in the $\mathrm{z}$ step and $\mathrm{z}$ tau scores. These results show that the CVR response is markedly faster in patients with concussion than in healthy controls. In patients with concussion, the white matter was more sensitive than the gray matter, with regard to the $\mathrm{z}$ step and $\mathrm{z}$ tau; this difference in the sensitivities of the white matter and gray matter is higher in patients with concussion than in healthy controls. The results show that the white matter is more easily damaged after concussion than the gray matter (Table 2). This indicates that the white matter shows more significant changes in CVR than the gray matter in patients with concussion.

\section{Discussion}

CVR is the expansion or contraction of blood vessels in response to stimulation. CVR is a reliable indicator for evaluating vascular reserve and self-regulating efficiency. CVR decline is related to normal aging (26) and is the most reliable neuroimaging predictor of impending cerebrovascular disease (28). Qualitative CVR information can be collected from the response of fMRI to any task (29), but to obtain quantitative CVR values, stimulants are usually required. Changes in intravascular $\mathrm{CO}_{2}$ can induce a strong $\mathrm{CBF}$ response, and $\mathrm{CO}_{2}$ inhalation is considered the best form of stimulation. Although the midbrain $\mathrm{CO}_{2}$ chemoreceptor can regulate respiration and blood flow, it also actively adjusts $\mathrm{CO}_{2}$-related blood $\mathrm{pH}$ to partially maintain homeostasis. Therefore, hypercapnia induced by increased arterial $\mathrm{CO}_{2}$ content activates intravascular potassium channels (30), resulting in a substantial increase in CBF, but no significant increase in metabolic rate (31).
Our previous research has shown that based on the expected goal of staged changes in $\mathrm{P}_{\mathrm{ET}} \mathrm{CO}_{2}$, the first to use an uneven task design: a rapid $\mathrm{CO}_{2}$ rise (Step) followed by a slow $\mathrm{CO}_{2}$ rise (Ramp) (32). People usually raise $\mathrm{P}_{\mathrm{ET}} \mathrm{CO}_{2}$ by $10 \mathrm{mmHg}$. The motivation for this design is to more accurately estimate the CVR response time and may reflect the amplitude of arterial response (33). In addition, both hypercapnia and hypocapnia are gradually induced by Step stimulation. It has been shown that the different segmentation of Ramp results in $\mathrm{P}_{\mathrm{ET}} \mathrm{CO}_{2}$ values of $30-50 \mathrm{mmHg}$, showing different spatial patterns in CVR, which can supplement traditional CVR information (34). This design uses both hypercapnia and hypocapnia for CVR estimation, making the deviation caused by the basal vascular tone more robust (35).

This study shows that CVR metrics have a high degree of sensitivity for detecting the presence of an acute concussion. To the best of our knowledge, their sensitivities and specificities exceed any other known detection methods. A previous study has demonstrated that acute and subacute concussion are associated with alterations in CVR that can be reliably detected by brain $\mathrm{MRI}-\mathrm{CO}_{2}$ stress testing in individual patients (36). Our results indicate that CVR response is larger and faster in patients with concussion than in healthy controls. Measurement of CVR using BOLD MRI and precisely controlled $\mathrm{CO}_{2}$ has been shown to be a safe, reliable, reproducible, and clinically useful method for the assessment of patients with concussion (37). MPET of $\mathrm{CO}_{2}$ applied to BOLD MRI yields superior spatial resolution and ability to evaluate the whole brain and specific regions compared to ASL (36).

Sobczyk et al. stated that compared with the reference map, the CVR of the corresponding voxel is scored according to its z-score by comparing with the reference atlas (25). This normalizes the voxel CVR score of the anatomical location and provides its probability score within 
the normal range. The size of the z-score (a larger number means a lower probability of normal response) is colorcoded and superimposed on the subject's anatomical scan, providing a comprehensive view of the normal and abnormal response distribution in the scan. Therefore, this CVR scoring technique takes into account the anatomical location of voxels and normal variability. This is especially important in the study of concussion, because the damage of concussion may be diffuse or isolated. Therefore, in our study, we chose the $\mathrm{z}$-score and $\mathrm{z}$ map as comparative indicators.

\section{CVR is increased in patients with concussion}

Our study shows that the CVR is increased in patients with concussion. Such changes may be related to the underlying pathophysiology (38). Evidence suggests that CVR increases within the first week after concussion, and this presumably indicates a disruption of normal autoregulation (36). This may be because of the dysregulation of the autonomic nervous system. This exaggerated response indicates damage to the normal cerebrovascular autoregulation function, which is worthy of further study. Interestingly, the shortened latency and increased amplitude of the CVR response were consistent with the symptoms of "physiological" concussion. People with concussions may experience migraine-like symptoms even during mild exercise, which explains the severe regional headaches they may experience. Differences in the mechanism of impact in patients with concussion results in heterogeneity among individuals. Consequently, it is difficult to identify common imaging biomarkers for different patients with concussion (39). A concussion is associated with inflammation, glymphatic clearance disorders, and cellular damage with physiological perturbations that may extend chronically beyond recovery (40). Glymphatic clearance is dependent on arterial blood flow (41), which may be affected by impaired autonomic regulation. Another possible reason for changes in CVR after concussion is inflammation. After a concussion, the autonomic system may show altered vascular myogenic tone and/or altered vagal, abnormal autonomic nervous system integration, inappropriate hemodynamic responses with reduced capacity to react to the needs of increased systemic metabolic demand, and/or abnormality of the sympathetic nervous system function (1). According to reports, although glial fibrillary acidic protein is a promising biomarker for concussion, its differential role is limited to 72 hours after injury (42). The extended utility of CVR indicators in concussion has clinical value, especially because patients with concussion usually do not seek medical treatment immediately. Several neuroimaging techniques, such as diffusion tensor imaging, BOLD, and ASL, have advanced our understanding of the effects of concussion on brain structure and function. However, individual responses to concussion and the pathophysiological processes of concussion are not clear. Because each person shows differences in anatomy, vulnerability to injury, mechanism of injury, and changes in abnormalities over time, concussion affects people differently (43). The imaging results in this study provide novel information about acute concussion and potential brain areas showing altered function.

\section{White matter is more sensitive than gray matter}

The most surprising aspect of our study was the increase in both the speed and magnitude of CVR and this effect was strongest in the white matter. Pathological conditions are usually characterized by reduced response to various neurovascular diseases, including arteriovenous malformations, moyamoya disease, sickle cell disease, and vascular dementia. DTI is also sensitive to early and chronic white matter changes in concussion, and preliminary evidence suggests that this imaging technique has prognostic value (44). White matter diffusivity differed between the groups in multiple white matter tracts, including the corpus callosum, cingulum bundle, and thalamic radiations (45). According to diffusion measurement indicators, microstructural white matter changes have been reported in studies of concussion intervals ranging from less than 24 hours to 6 months (27). CVR may serve as an imaging biomarker to detect subtle deficits in both the gray matter and white matter for the diagnosis of individual concussions and help find the relationship between changes in the brain and symptoms manifesting after a concussion. The findings encourage further investigation of hypercapnic BOLD as a diagnostic tool for concussion (46). A concussion is associated with abnormal white matter microstructure and poor neurocognitive performance (47). Acute effects of concussion are associated with both hyperconnectivity and hypoconnectivity, with a disruption of white matter integrity (48).

\section{Reason for increased magnitude and speed of response in patients with acute concussion}

The increase in the magnitude and speed of CVR in 
patients with an acute concussion is difficult to explain as it represents a supernormal response to the vasodilatory stimulus. In order to better understand this contradictory response, the role of glial cells in regulating "neurovascular coupling" in the brain should be considered. Selective stimulation of glial cells can trigger vasoconstriction and/or dilatation (49). In light of current concepts of gliovascular and neurovascular coupling, disruption of the gliovascular modulation by physical injury may interrupt the "braking" system of blood flow control. Interestingly, glial-driven vascular responses have the characteristics of "large amplitude" and "short latency" and are considered to be direct glial-vascular signal transmission functions without the need for neurons as intermediaries (49). An increase in $\mathrm{O}_{2}$ delivery could result in additional injury via free radical toxicity. Proper regulation of $\mathrm{CBF}$ is critical for brain health and survival. CBF is precisely regulated by the neurovascular unit to ensure brain energy demands are met (50). Neurovascular coupling mechanisms increase the flow of blood to regions showing neuronal activity to sustain neuronal function (51). The pathological mechanism of concussion may involve damage to the NVU, which results in dysregulated $\mathrm{CBF}$ and neurovascular uncoupling.

\section{Limitations}

BOLD has advantages over positron emission tomography. It has greater time and spatial resolution than ASL, provides greater coverage, requires a shorter scan time, and is a sequence more commonly available on clinical scanners. However, specific artifacts are associated with BOLD, such as those arising from differences in baseline metabolism and alterations in cerebral blood volume (such as increased signal changes over venous pools of blood). Blood pressure is a key confounding factor, which affects the interpretation of CVR (39). However, in these experiments, we were limited by constraints imposed by the MRI environment on blood pressure measurement.

\section{Conclusions}

This study demonstrated that the measurement of CVR using BOLD MRI and precisely controlled $\mathrm{CO}_{2}$ is a safe, reliable, reproducible, and clinically useful method for patients with concussion. Our findings suggest that concussion is associated with patient-specific abnormalities in BOLD- $\mathrm{CO}_{2}$ cerebrovascular responsiveness that occur in the setting of the global CVR atlas. This study demonstrated that $\mathrm{z}$ step and $\mathrm{z}$ tau CVR could be validated and serve as objective biomarkers for the diagnosis or prediction of outcomes of concussion, which can facilitate evidence-based treatment of patients with concussion, especially targeting the white matter regions of the brain. Furthermore, the present study provides novel insights into the relationship between concussion and white matter $\mathrm{z}$ step CVR. Overall, this method holds great potential and promise for the prognosis of concussion and prediction of possible symptoms, because it is cost-effective, noninvasive, and highly sensitive in detecting CVR. It has the potential to be an important tool for assessing the severity and duration of symptoms after concussion and concussion diagnosis.

\section{Acknowledgments}

Funding: This research was supported by the National Key Research and Development Program of China (Grant No.: 2016YFC1305900) and the National Science Foundation of China (Grant No.: 31870981, 82020108016).

\section{Footnote}

Conflicts of Interest: All authors have completed the ICMJE uniform disclosure form (available at https://dx.doi. org/10.21037/qims-20-1296). The authors have no conflicts of interest to declare.

Ethical Statement: The authors are accountable for all aspects of the work in ensuring that questions related to the accuracy or integrity of any part of the work are appropriately investigated and resolved. The study protocol was designed in line with the guidelines outlined in the Declaration of Helsinki (as revised in 2013) and approved by the Research Ethics Board (REB) at the University Health Network in Toronto. After providing a complete description of the study, informed written consent was obtained from each participant before the study.

Open Access Statement: This is an Open Access article distributed in accordance with the Creative Commons Attribution-NonCommercial-NoDerivs 4.0 International License (CC BY-NC-ND 4.0), which permits the noncommercial replication and distribution of the article with the strict proviso that no changes or edits are made and the original work is properly cited (including links to both the formal publication through the relevant DOI and the license). 
See: https://creativecommons.org/licenses/by-nc-nd/4.0/.

\section{References}

1. Tasker RC. Anesthesia and concussion. Curr Opin Anaesthesiol 2017;30:343-8.

2. Thomas RE, Alves J, Vaska Mlis MM, Magalhaes R. Therapy and rehabilitation of mild brain injury/ concussion: Systematic review. Restor Neurol Neurosci 2017;35:643-66.

3. Zuckerman SL, Brett BL, Jeckell AS, Yengo-Kahn AM, Solomon GS. Prognostic Factors in Pediatric Sport-Related Concussion. Curr Neurol Neurosci Rep 2018;18:104.

4. Doshi H, Wiseman N, Liu J, Wang W, Welch RD, O'Neil BJ, Zuk C, Wang X, Mika V, Szaflarski JP, Haacke EM, Kou Z. Cerebral hemodynamic changes of mild traumatic brain injury at the acute stage. PLoS One 2015;10:e0118061.

5. Boshra R, Dhindsa K, Boursalie O, Ruiter KI, Sonnadara R, Samavi R, Doyle TE, Reilly JP, Connolly JF. From GroupLevel Statistics to Single-Subject Prediction: Machine Learning Detection of Concussion in Retired Athletes. IEEE Trans Neural Syst Rehabil Eng 2019;27:1492-501.

6. Shafi R, Poublanc J, Venkatraghavan L, Crawley AP, Sobczyk O, McKetton L, Bayley M, Chandra T, Foster E, Ruttan L, Comper P, Tartaglia MC, Tator CH, Duffin J, Mutch WA, Fisher J, Mikulis DJ. A Promising SubjectLevel Classification Model for Acute Concussion Based on Cerebrovascular Reactivity Metrics. J Neurotrauma 2021;38:1036-47.

7. Iadecola C. The Neurovascular Unit Coming of Age: A Journey through Neurovascular Coupling in Health and Disease. Neuron 2017;96:17-42.

8. Churchill NW, Hutchison MG, Graham SJ, Schweizer TA. Baseline vs. cross-sectional MRI of concussion: distinct brain patterns in white matter and cerebral blood flow. Sci Rep 2020;10:1643.

9. Leung J, Kosinski PD, Croal PL, Kassner A. Developmental trajectories of cerebrovascular reactivity in healthy children and young adults assessed with magnetic resonance imaging. J Physiol 2016;594:2681-9.

10. Nzou G, Wicks RT, VanOstrand NR, Mekky GA, Seale SA, El-Taibany A, Wicks EE, Nechtman CM, Marrotte EJ, Makani VS, Murphy SV, Seeds MC, Jackson JD, Atala AJ. Multicellular 3D Neurovascular Unit Model for Assessing Hypoxia and Neuroinflammation Induced Blood-Brain Barrier Dysfunction. Sci Rep 2020;10:9766.
11. Fisher JA. The $\mathrm{CO} 2$ stimulus for cerebrovascular reactivity: Fixing inspired concentrations vs. targeting end-tidal partial pressures. J Cereb Blood Flow Metab 2016;36:1004-11.

12. Duffin J, Sobczyk O, Crawley A, Poublanc J, Venkatraghavan L, Sam K, Mutch A, Mikulis D, Fisher J. The role of vascular resistance in BOLD responses to progressive hypercapnia. Hum Brain Mapp 2017;38:5590-602.

13. Mandell DM, Han JS, Poublanc J, Crawley AP, Fierstra J, Tymianski M, Fisher JA, Mikulis DJ. Quantitative measurement of cerebrovascular reactivity by blood oxygen level-dependent MR imaging in patients with intracranial stenosis: preoperative cerebrovascular reactivity predicts the effect of extracranial-intracranial bypass surgery. AJNR Am J Neuroradiol 2011;32:721-7.

14. Ellis MJ, Ryner LN, Sobczyk O, Fierstra J, Mikulis DJ, Fisher JA, Duffin J, Mutch WA. Neuroimaging Assessment of Cerebrovascular Reactivity in Concussion: Current Concepts, Methodological Considerations, and Review of the Literature. Front Neurol 2016;7:61.

15. da Costa L, van Niftrik CB, Crane D, Fierstra J, Bethune A. Temporal Profile of Cerebrovascular Reactivity Impairment, Gray Matter Volumes, and Persistent Symptoms after Mild Traumatic Head Injury. Front Neurol 2016;7:70.

16. Tagge CA, Fisher AM, Minaeva OV, GaudreauBalderrama A, Moncaster JA, Zhang XL, et al. Concussion, microvascular injury, and early tauopathy in young athletes after impact head injury and an impact concussion mouse model. Brain 2018;141:422-58.

17. Giza C, Greco T, Prins ML. Concussion: pathophysiology and clinical translation. Handb Clin Neurol 2018;158:51-61.

18. Wang Y, Nencka AS, Meier TB, Guskiewicz K, Mihalik JP, Alison Brooks M, Saykin AJ, Koch KM, Wu YC, Nelson LD, McAllister TW, Broglio SP, McCrea MA. Cerebral blood flow in acute concussion: preliminary ASL findings from the NCAA-DoD CARE consortium. Brain Imaging Behav 2019;13:1375-85.

19. Smith KJ, Ainslie PN. Regulation of cerebral blood flow and metabolism during exercise. Exp Physiol 2017;102:1356-71.

20. McKetton L, Sobczyk O, Duffin J, Poublanc J, Sam K, Crawley AP, Venkatraghavan L, Fisher JA, Mikulis DJ. The aging brain and cerebrovascular reactivity. Neuroimage 2018;181:132-41.

21. Poublanc J, Crawley AP, Sobczyk O, Montandon G, Sam 
K, Mandell DM, Dufort P, Venkatraghavan L, Duffin J, Mikulis DJ, Fisher JA. Measuring cerebrovascular reactivity: the dynamic response to a step hypercapnic stimulus. J Cereb Blood Flow Metab 2015;35:1746-56.

22. Ito S, Mardimae A, Han J, Duffin J, Wells G, Fedorko L, Minkovich L, Katznelson R, Meineri M, Arenovich T, Kessler C, Fisher JA. Non-invasive prospective targeting of arterial $\mathrm{P}(\mathrm{CO} 2)$ in subjects at rest. J Physiol 2008;586:3675-82.

23. Somogyi RB, Vesely AE, Preiss D, Prisman E, Volgyesi G, Azami T, Iscoe S, Fisher JA, Sasano H. Precise control of end-tidal carbon dioxide levels using sequential rebreathing circuits. Anaesth Intensive Care 2005;33:726-32.

24. Willie CK, Macleod DB, Shaw AD, Smith KJ, Tzeng YC, Eves ND, Ikeda K, Graham J, Lewis NC, Day TA, Ainslie PN. Regional brain blood flow in man during acute changes in arterial blood gases. J Physiol 2012;590:3261-75.

25. Sobczyk O, Battisti-Charbonney A, Poublanc J, Crawley AP, Sam K, Fierstra J, Mandell DM, Mikulis DJ, Duffin J, Fisher JA. Assessing cerebrovascular reactivity abnormality by comparison to a reference atlas. J Cereb Blood Flow Metab 2015;35:213-20.

26. Teuho J, Linden J, Johansson J, Tuisku J, Tuokkola T, Teras M. Tissue probability-based AC for neurological PET/MR using SPM8. EJNMMI Phys 2015;2:A26.

27. Lu H, Xu F, Rodrigue KM, Kennedy KM, Cheng Y, Flicker B, Hebrank AC, Uh J, Park DC. Alterations in cerebral metabolic rate and blood supply across the adult lifespan. Cereb Cortex 2011;21:1426-34.

28. Pillai JJ, Mikulis DJ. Cerebrovascular reactivity mapping: an evolving standard for clinical functional imaging. AJNR Am J Neuroradiol 2015;36:7-13.

29. Dumas A, Dierksen GA, Gurol ME, Halpin A, MartinezRamirez S, Schwab K, Rosand J, Viswanathan A, Salat DH, Polimeni JR, Greenberg SM. Functional magnetic resonance imaging detection of vascular reactivity in cerebral amyloid angiopathy. Ann Neurol 2012;72:76-81.

30. Ainslie PN, Duffin J. Integration of cerebrovascular $\mathrm{CO} 2$ reactivity and chemoreflex control of breathing: mechanisms of regulation, measurement, and interpretation. Am J Physiol Regul Integr Comp Physiol 2009;296:R1473-95.

31. Chen JJ, Pike GB. Global cerebral oxidative metabolism during hypercapnia and hypocapnia in humans: implications for BOLD fMRI. J Cereb Blood Flow Metab 2010;30:1094-9.
32. Spano VR, Mandell DM, Poublanc J, Sam K, BattistiCharbonney A, Pucci O, Han JS, Crawley AP, Fisher JA, Mikulis DJ. CO2 blood oxygen level-dependent MR mapping of cerebrovascular reserve in a clinical population: safety, tolerability, and technical feasibility. Radiology 2013;266:592-8.

33. Duffin J, Sobczyk O, Crawley AP, Poublanc J, Mikulis DJ, Fisher JA. The dynamics of cerebrovascular reactivity shown with transfer function analysis. Neuroimage 2015;114:207-16.

34. Fisher JA, Sobczyk O, Crawley A, Poublanc J, Dufort P, Venkatraghavan L, Sam K, Mikulis D, Duffin J. Assessing cerebrovascular reactivity by the pattern of response to progressive hypercapnia. Hum Brain Mapp 2017;38:3415-27.

35. Halani S, Kwinta JB, Golestani AM, Khatamian YB, Chen JJ. Comparing cerebrovascular reactivity measured using BOLD and cerebral blood flow MRI: The effect of basal vascular tension on vasodilatory and vasoconstrictive reactivity. Neuroimage 2015;110:110-23.

36. Mutch WAC, Ellis MJ, Ryner LN, McDonald PJ, Morissette MP, Pries P, Essig M, Mikulis DJ, Duffin J, Fisher JA. Patient-Specific Alterations in CO2 Cerebrovascular Responsiveness in Acute and Sub-Acute Sports-Related Concussion. Front Neurol 2018;9:23.

37. McKetton L, Venkatraghavan L, Poublanc J, Sobczyk O, Crawley AP, Rosen C, Silver FL, Duffin J, Fisher JA, Mikulis DJ. Importance of Collateralization in Patients With Large Artery Intracranial Occlusive Disease: LongTerm Longitudinal Assessment of Cerebral Hemodynamic Function. Front Neurol 2018;9:226.

38. Sobczyk O, Battisti-Charbonney A, Fierstra J, Mandell DM, Poublanc J, Crawley AP, Mikulis DJ, Duffin J, Fisher JA. A conceptual model for $\mathrm{CO}_{2}$-induced redistribution of cerebral blood flow with experimental confirmation using BOLD MRI. Neuroimage 2014;92:56-68.

39. Gioia GA, Collins M, Isquith PK. Improving identification and diagnosis of mild traumatic brain injury with evidence: psychometric support for the acute concussion evaluation. J Head Trauma Rehabil 2008;23:230-42.

40. Di Battista AP, Churchill N, Schweizer TA, Rhind SG, Richards D, Baker AJ, Hutchison MG. Blood biomarkers are associated with brain function and blood flow following sport concussion. J Neuroimmunol 2018;319:1-8.

41. Iliff JJ, Wang M, Zeppenfeld DM, Venkataraman A, Plog BA, Liao Y, Deane R, Nedergaard M. Cerebral arterial pulsation drives paravascular CSF-interstitial fluid exchange in the murine brain. J Neurosci 
2013;33:18190-9.

42. Papa L, Zonfrillo MR, Welch RD, Lewis LM, Braga CF, Tan CN, Ameli NJ, Lopez MA, Haeussler CA, Mendez Giordano D, Giordano PA, Ramirez J, Mittal MK. Evaluating glial and neuronal blood biomarkers GFAP and UCH-L1 as gradients of brain injury in concussive, subconcussive and non-concussive trauma: a prospective cohort study. BMJ Paediatr Open 2019;3:e000473.

43. $\mathrm{Mu} \mathrm{W}$, Catenaccio E, Lipton ML. Neuroimaging in BlastRelated Mild Traumatic Brain Injury. J Head Trauma Rehabil 2017;32:55-69.

44. Meier TB, Bergamino M, Bellgowan PS, Teague TK, Ling JM, Jeromin A, Mayer AR. Longitudinal assessment of white matter abnormalities following sports-related concussion. Hum Brain Mapp 2016;37:833-45.

45. Wilde EA, Goodrich-Hunsaker NJ, Ware AL, Taylor BA, Biekman BD, Hunter JV, Newman-Norlund R, Scarneo S, Casa DJ, Levin HS. Diffusion Tensor Imaging Indicators of White Matter Injury Are Correlated with a Multimodal Electroencephalography-Based Biomarker in Slow Recovering, Concussed Collegiate Athletes. J Neurotrauma 2020;37:2093-101.

Cite this article as: Wang $\mathrm{R}$, Poublanc J, Crawley AP, Sobczyk O, Kneepkens S, Mcketton L, Tator C, Wu R, Mikulis DJ. Cerebrovascular reactivity changes in acute concussion: a controlled cohort study. Quant Imaging Med Surg 2021;11(11):4530-4542. doi: 10.21037/qims-20-1296
46. Chan ST, Evans KC, Rosen BR, Song TY, Kwong KK. A case study of magnetic resonance imaging of cerebrovascular reactivity: a powerful imaging marker for mild traumatic brain injury. Brain Inj 2015;29:403-7.

47. Lipton ML, Kim N, Zimmerman ME, Kim M, Stewart WF, Branch CA, Lipton RB. Soccer heading is associated with white matter microstructural and cognitive abnormalities. Radiology 2013;268:850-7.

48. Murdaugh DL, King TZ, Sun B, Jones RA, Ono KE, Reisner A, Burns TG. Longitudinal Changes in Resting State Connectivity and White Matter Integrity in Adolescents With Sports-Related Concussion. J Int Neuropsychol Soc 2018;24:781-92.

49. Attwell D, Buchan AM, Charpak S, Lauritzen M, Macvicar BA, Newman EA. Glial and neuronal control of brain blood flow. Nature 2010;468:232-43.

50. Zlokovic BV. Neurovascular pathways to neurodegeneration in Alzheimer's disease and other disorders. Nat Rev Neurosci 2011;12:723-38.

51. Metea MR, Newman EA. Glial cells dilate and constrict blood vessels: a mechanism of neurovascular coupling. J Neurosci 2006;26:2862-70. 